\title{
ELECTRODYNAMIC LOUDSPEAKER-DRIVEN ACOUSTIC COMPRESSOR
}

\begin{abstract}
Martin Šoltés*, Milan Červenka
Czech Technical University in Prague, Faculty of Electrical Engineering, Technická 2, Prague, Czech Republic

* corresponding author: soltes.mail@gmail.com

ABstract. An acoustic compressor is built using the acoustic resonator which shape was optimized for a maximum acoustic pressure amplitude and a low-cost compression driver. Acoustic compressor is built by installing a suction port in the resonator wall where the standing wave has its pressure node and a delivery port with a valve in the resonator wall where the standing wave has its pressure anti-node. Different reeds, serving as delivery valves, are tested and their performance is investigated. It was shown that the performance of such a simple compressor is comparable or better than the acoustic compressors built previously by other researchers using non-optimally shaped resonators with more sophisticated driving mechanisms and valve arrangements.
\end{abstract}

KEYWORDS: acoustic compressor, acoustic resonator, high amplitude acoustic field.

\section{INTRODUCTION}

High amplitude standing waves in closed cavities have been studied extensively by many researchers (see e.g. 1-4, for the review of the research done on the subject up to 1996, see [5]). Much of the effort has been devoted to the study of the special case of piston-driven constant cross-section resonators driven at or close to one of its resonance frequencies. However, no matter how strong the excitation, the maximum acoustic pressure amplitudes obtained in the constant cross-section resonators were limited. It has been observed and is now a well-known fact that when resonators of cylindrical shape are excited at their resonant frequency, acoustic energy is transferred from the fundamental resonance to higher harmonics due to a non-linear properties of fluid, eventually leading to a formation of the shock wave. Due to the fact, that dissipation of acoustic energy is proportional, the shock formation sets the upper limit for the maximum acoustic pressure in such resonator.

Gaitan and Atchley [3] showed that it is possible to prevent the formation of shock waves in tubes with variable cross-section, where energy transfer from the fundamental resonance to the higher resonance modes is significantly reduced.

In 1998, Lawrenson et al. 4 published their experimental paper in which they introduced the concept of Resonant Macrosonic Synthesis (RMS). They showed that relative phases and amplitudes of the harmonics can be controlled by the resonator geometry resulting in shock-free waveforms of extremely high amplitudes. Acoustic pressure amplitudes that they obtained were more than an order of magnitude larger than it had been possible before.

Ilinskii et al. 6] presented a one-dimensional nonlinear model equation for description of high-amplitude standing waves in axi-symmetric, arbitrarily shaped acoustic resonators in their theoretical paper.

Li et al. [] presented a method for optimization of the resonator shapes based on a nonlinear wave equation. They considered resonator shapes described by smooth elementary functions with adjustable parameters only.

Červenka et al. 8] proposed an evolutionary algorithm-based method for optimizing the shape of the acoustic resonators for achieving high-amplitude shock-free acoustic fields. The method is based on a linear model that includes losses due to turbulence in the boundary layer. They used a more general approach of parametrizing the resonator shape using control points interconnected with cubic splines.

With the progress in both theoretical description as well as experimental results in the field of highamplitude acoustic fields a number of different practical applications emerged, e.g. acoustic compressors, plasma-chemical reactors 9], thermoacoustic devices [10, etc.

The possibility of constructing an acoustic compressor has been investigated by several authors (Bodine [11, Lucas [12, El-Sabbagh [13, Masuda and Kawashima [14] and Hossain et al. [15]). Acoustic compressors offer several advantages over the more traditional ones. Most importantly they do not contain moving parts that require oils to reduce friction and wear - this is important in applications where mixing of oil with the compressed fluid is undesirable. Moving parts also reduce reliability of the compressor since they are subject to mechanical fatigue and failure. Another advantage is that acoustic compressors allow using a valveless construction [12. In all of the experiments researches used either the piston-driven resonators or the entirelydriven resonators (resonators in which acoustic energy is introduced in the resonator cavity by shaking the whole resonator along its axis).

In this paper we aim to experimentally demonstrate practical utilisation of high-amplitude acoustic fields made possible by the optimized resonator described in our previous paper [8] by constructing a working exam- 


\begin{tabular}{rccrrrrrrrr}
\hline$x[\mathrm{~mm}]$ & 0 & 33.3 & 66.7 & 100 & 133.3 & 166.7 & 200 & 233.3 & 266.7 & 300 \\
\hline$r[\mathrm{~mm}]$ & 5 & 5 & 5 & 5.4 & 7.6 & 10.6 & 15.1 & 23.1 & 25 & 25 \\
\hline
\end{tabular}

TABLE 1. Coordinates of the ten control points for cubic-spline interpolation describing the shape of the resonator used in experiments.

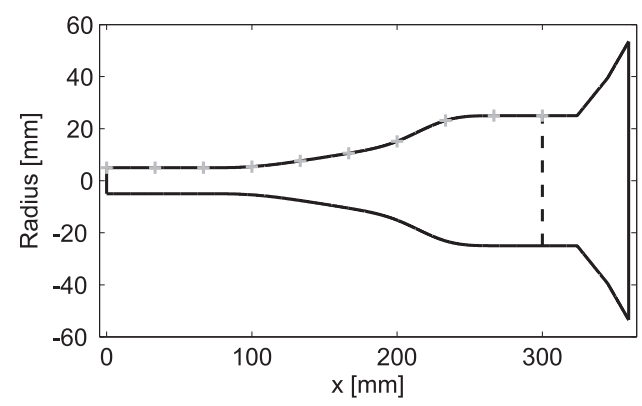

Figure 1. Shape of the resonator cavity. Grey crosses denote positions of the ten control points for cubicspline interpolation. Part of the resonator to the right of the dashed line represents the internal waveguide inside the Selenium driver. Resonator is terminated by a rigid wall on its narrow end (at $x=0 \mathrm{~mm}$ ) and by the loudspeaker diaphragm on its wide end (at $x=l_{\mathrm{t}}$ ).

ple of an acoustic compressor. We use the commonly available loudspeaker-driver to excite the resonator since it is much cheaper and smaller compared with the traditionally used electromagnetic or electrodynamic shakers to increase the economic attractiveness of the proposed solution.

\section{Acoustic COMPRESSOR CONSTRUCTION}

The experiments were conducted on the system consisting of electrodynamic loudspeaker and external resonator cavity. The compression driver Selenium DT-405Ti, from which the phase-plug was removed, was used along with a $300 \mathrm{~mm}$ long external resonator ${ }^{1}$ External resonator, machined from two pieces of duralumin block which were joined together, has an axisymmetrical cavity, which shape was optimized for the use with the Selenium loudspeaker for maximal acoustic pressure amplitude at the closed end of the resonator (at $x=0 \mathrm{~mm}$ ).

The optimization procedure, described in our previous paper [8] is an evolutionary strategy-based method that optimizes the shapes of resonators subject to a given set of constraints (minimum and maximum radius, minimum and maximum resonance frequency, resonator length). Resonator shape is defined by a set of $N$ control-points (serving as parameters for the optimization) and is obtained by a cubic-spline interpolation. We have used $N=10$ control points and

\footnotetext{
${ }^{1}$ Resonating cavity of the whole system is formed by the short internal waveguide inside the driver and external resonator attached to the driver. Total resonator length $l_{\mathrm{t}}$ is therefore given by $l_{\mathrm{t}}=l_{\text {int }}+l_{\text {ext }}$, where $l_{\text {int }}$ is the length of the internal waveguide and $l_{\text {ext }}$ is the length of the external waveguide.
}

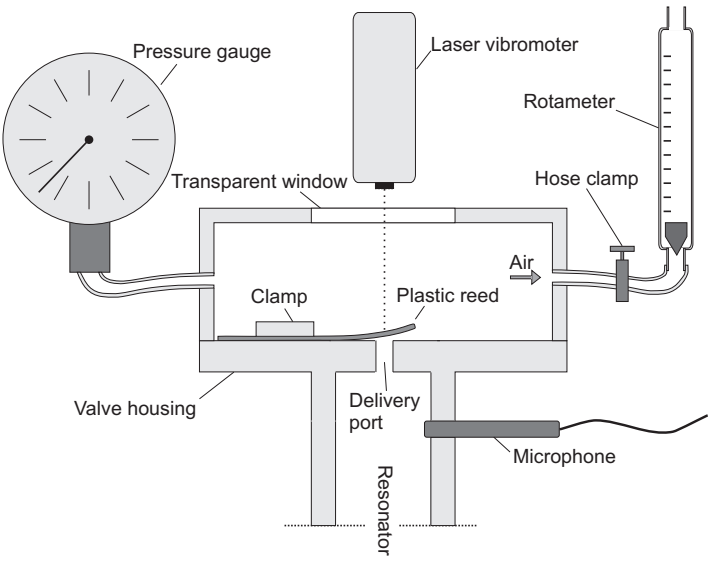

FIGURE 2. Valve housing configuration.

the following constraints: $r_{\min }=5 \mathrm{~mm}, r_{\max }=25 \mathrm{~mm}$, $l_{\text {ext }}=300 \mathrm{~mm}$ in the optimization procedure. Resonance frequency constraints were not applied. The resulting resonator shape is shown in Figure 1. Coordinates of the control points (shown as gray crosses in Figure 1) are listed in Table 1

The acoustic compressor was built by installing a suction port in the resonator wall where standing wave has its pressure node (at $x=272 \mathrm{~mm}$ from the closed end of the resonator in our case) and a delivery port in the resonator wall where the standing wave has its anti-node (at $x=0 \mathrm{~mm}$ ).

With this arrangement alone, it is already possible to produce one-way air flow ${ }^{2}[12$, however the value of the air flow-rate obtained this way is very small. For this reason the delivery port was fitted with a valve which rectifies the flow of the medium and therefore allows much higher values of air flow-rate to be achieved. In our experiments we have used a reed-type passive valve due to its simplicity and, more importantly, due to its ability to operate at high frequencies. The valve opens when acoustic pressure inside the resonator cavity rises above the static ambient pressure at the other side of the reed and closes when the acoustic pressure falls bellow this static pressure level resulting in one-way air flow.

Delivery port - a circular hole of $4 \mathrm{~mm}$ diameter with the center at resonator axis of symmetry was drilled in the cap closing the resonator at its narrow end (at $x=0 \mathrm{~mm}$ ). Delivery valve, enclosed inside a small metal box, was installed in a way shown in

\footnotetext{
${ }^{2}$ This is possible due to nonlinear properties of a fluid - with rising acoustic pressure amplitude inside the resonator, some small dc component emerges at the pressure anti-node. This creates static pressure gradient inside the resonator causing air flow between suction and delivery port.
} 


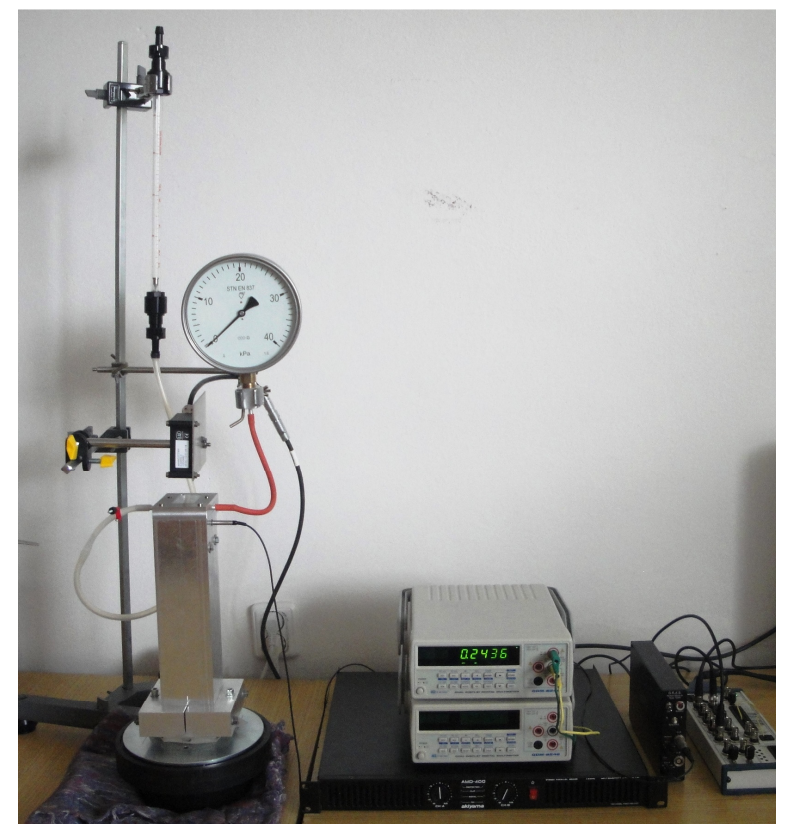

FIGURE 3. The acoustic compressor experimental setup. The selenium driver with the resonator attached to it is in the left part of the picture. The delivery valve housing is attached on the top of the resonator and laser vibrometer is placed above. One of the hoses connects the pressure gauge and the other one with a hose clamp leads to the flow-meter. A microphone is connected from the side of the resonator.

Figure 2. This box (delivery valve housing) features two outlets for connecting the pressure gauge and air-flow meter. It also features a transparent glass window in its top wall, which can be used for the measurement of the reed displacement using a laser vibrometer. Two holes of $2 \mathrm{~mm}$ diameter drilled in the opposite sides of the resonator wall at the pressure node serve as the suction ports.

\section{Results}

\subsection{EXPERIMENTAL SETUP}

All measurements were performed at a room temperature and atmospheric pressure using air as a compressed medium. The electrical signal driving the loudspeaker was generated with a computer using the LabVIEW environment and amplified with the Akiyama AMD400 amplifier. Acoustic pressure measurements were made in the LabVIEW environment using the NI PCI-6251 data acquisition card and the G.R.A.S. 12AA preamplifier with the G.R.A.S. 40DP 1/8" microphone which was attached from the side of the resonator as shown in Figure 2 Static pressure was measured using the PTL Prematlak 2010 pressure gauge, air volume flow-rate was measured using the Rheotest Medingen PG05 rotameter and the $\mu \epsilon$ optoNCDT ILD2300 laser vibrometer was used for the reed displacement measurement. Figure 3 shows a photograph of the experimental setup.

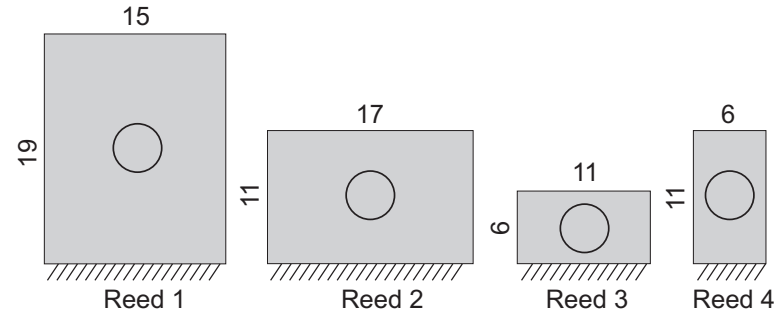

Figure 4 . The valve reeds used in the experiment. Dimensions are in millimetres. Circles in the middle of each reed denote the position of the delivery port.

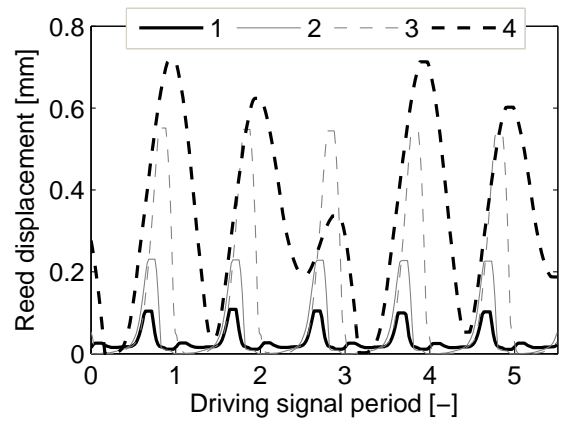

Figure 5. Measurement of the displacement versus the time characteristic for four different valve reeds shown in Figure 4 The displacement was measured with the laser beam pointing at the center of the delivery port.

\subsection{Static PRESSURE With NO FLOW}

When the acoustic pressure is present inside the resonator, the delivery valve acts as a rectifier - increasing static pressure on the other side of the valve. By closing the hose clamp that controls the air flow (see Figure 2) air is prevented from leaving the delivery valve housing resulting in the static pressure build-up. Since there is no air-flow out of the delivery valve housing, optimally performing valve would eventually produce static pressure (inside the delivery valve housing) equal to the acoustic pressure amplitude (inside the resonator). Displacement of the reed should decrease with the rising static pressure and the reed should eventually stop vibrating once the steady-state is reached - when the static pressure reaches the value of the acoustic pressure amplitude. This is not possible in our simple arrangement where the reed is not allowed to vibrate symmetrically but we can expect better performing reeds having smaller displacement.

A number of different valve reeds were tried and it was found that dimensions of the reed have a significant impact on its performance. Results for four different reeds shown in Figure 4 are presented below. All reeds were made from a $130 \mu \mathrm{m}$ thick PVC foil.

Figure 5 shows a measurement of the steady-state reed displacement versus the time for four different reeds shown in Figure 4 The resonator was driven with sine-wave signal at resonance frequency $f_{\text {res }}=551 \mathrm{~Hz}$ with the input voltage amplitude $\left|U_{\text {in }}\right|=10 \mathrm{~V}$. It can be observed that the valve reed 1 tracks the acoustic pressure well. It opens only for a short interval during 


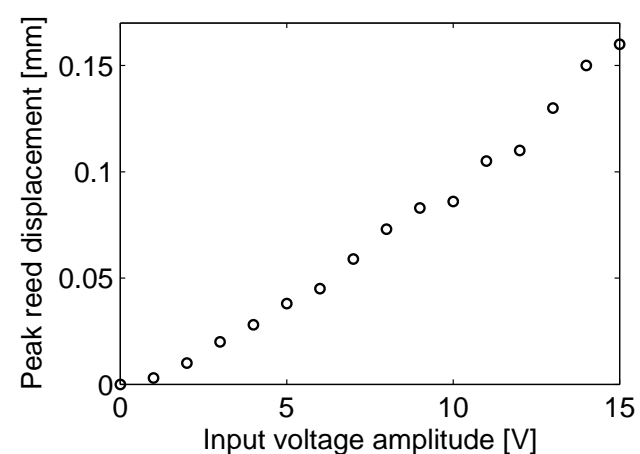

Figure 6 . The valve reed 1 displacement amplitude versus input voltage characteristic.

\begin{tabular}{lcccc}
\hline Reed number & 1 & 2 & 3 & 4 \\
\hline Pressure [kPa] & 20 & 16 & 7 & 0
\end{tabular}

TABLE 2. Static pressures measured inside the delivery valve housing, with the resonator driven at the resonance with the input voltage amplitude $\left|U_{\text {in }}\right|=10 \mathrm{~V}$.

each cycle with the maximum displacement around $0.1 \mathrm{~mm}$. Valve reed 4 does not track the acoustic pressure very well. It is open during most of the cycle and its maximum displacement is roughly seven times bigger than the maximum displacement of the valve reed 1 . Valve reeds 2 and 3 behave somewhere between these two extremes.

The static pressures inside the delivery valve housing measured at these conditions are summarized in the Table 2 .

It is clear that the valve reeds which behave closer to the ideal - exhibiting smaller displacement, which are able to track the acoustic pressure more accurately (i.e. respond faster) produce higher static pressures. We use the valve reed 1 in all of the experiments described below.

Figure 6 6 shows the valve displacement versus the input voltage characteristic. It can be observed that the valve reed displacement grows roughly proportionately with the input voltage suggesting a good - linear behaviour of the valve.

Figure 7 shows the measured static pressure and the acoustic pressure amplitude versus the input voltage characteristic. It can be observed that the system possesses the desired characteristic - most of the acoustic pressure amplitude is rectified into the static pressure.

\subsection{RESONATOR WITH AIR FLOW}

By gradually releasing the hose clamp, the air is allowed to escape the valve housing that results in the decrease of static (delivery) pressure and one-way air flow through the resonator. Figure 8 shows the air volume flow-rate versus the delivery pressure characteristic measured at three different input voltage amplitudes.

It was observed that the frequency at which maximum air flow is achieved increases with opening of

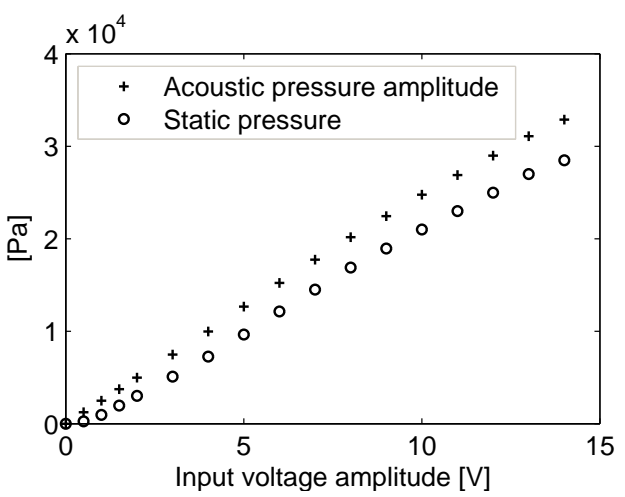

FIgURE 7 . The acoustic pressure amplitude inside the resonator measured near the delivery port (as shown in Figure 2 and the static pressure measured inside the delivery valve housing versus the input voltage characteristic.

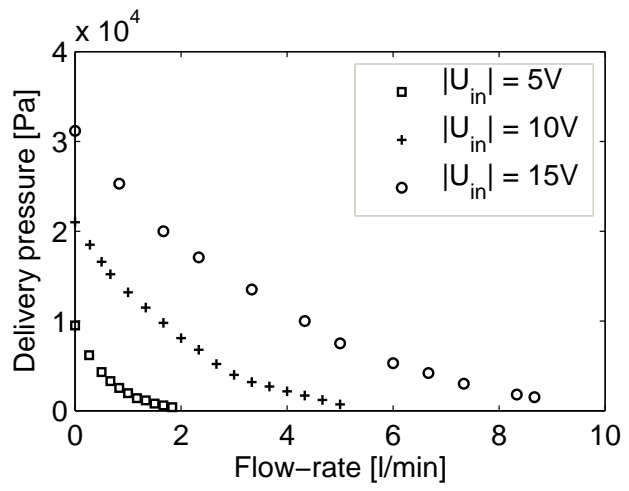

Figure 8. The air volume flow-rate versus the delivery pressure measured for the three different input voltage amplitudes.

the hose clamp. However, it does not depend on the absolute value of the air flow rate or on the delivery pressure. Figure 9 illustrates this frequency shift. The vertical axis represents frequency at which air flow is maximal (for a given hose clamp setting) while the horizontal axis represents this air flow rate as a percentage of the maximum possible air flow rate - with the hose clamp removed. This is measured for three different input voltage amplitudes. In other words, Figure 9 illustrates how air flow rate and frequency at which air flow rate is maximal changes with opening of the hose clamp. It can be observed that the frequency shifts similarly (in relative terms) for all three input voltages - irrespective of the absolute air flow rate or static pressure. It seems that the frequency at which the air flow rate is maximal depends only on how much the hose clamp is opened. Possible explanation of this behaviour is that as the clamp is opened the effective geometry of the cavity behind the delivery valve changes. Since this cavity itself forms an acoustic system the change in its geometry could affect the function of the delivery valve. Similar behaviour was also observed by Masuda and Kawashima [14. 


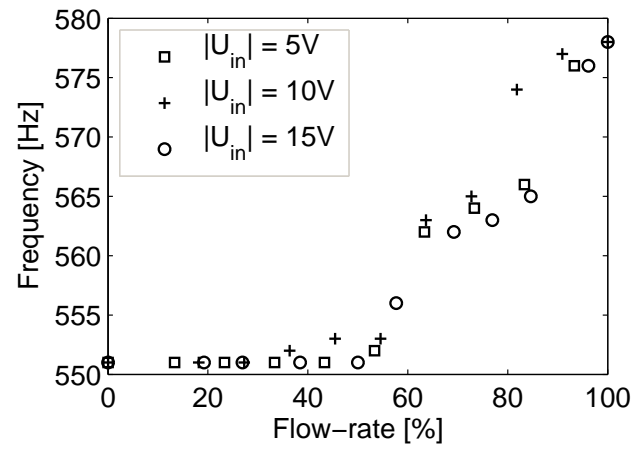

Figure 9. The shift of the driving signal frequency at which the air flow-rate is maximal as the hose clamp is opened. Description of the figure is given in the text above.

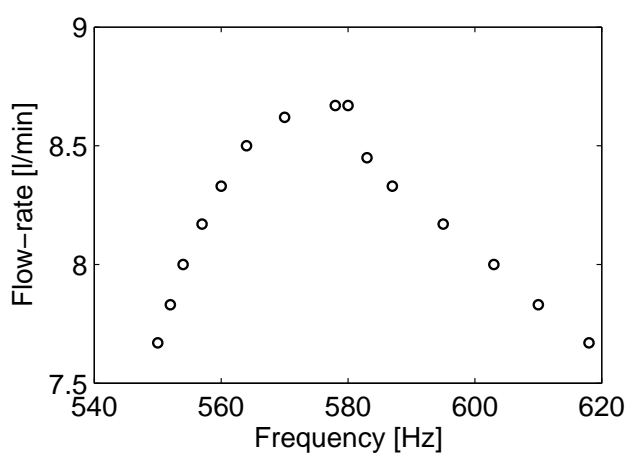

Figure 10. Frequency characteristic of the air volume flow-rate at input voltage $\left|U_{\text {in }}\right|=15 \mathrm{~V}$ with the hose clamp removed.

The measured frequency characteristic of the air volume flow-rate at the input voltage $\left|U_{\text {in }}\right|=15 \mathrm{~V}$ with the hose clamp removed is shown in Figure 10 It can be observed that under these conditions adjusting the driving frequency from the $f_{\text {res }}=551 \mathrm{~Hz}$ to $f=580 \mathrm{~Hz}$ results in roughly $14 \%$ increase of the air volume flowrate.

\section{Conclusions}

We have shown that using the optimized resonator described in our previous paper [8] construction of acoustic compressor is possible even when using a relatively inexpensive and simple driving mechanism - a compression driver. Air volume flow-rates and delivery pressures we have been able to achieve (Figure 8) are comparable or better than the ones reported by other authors [13-15].

It was observed that the dimensions of the reed, which acts as a delivery valve, has a significant impact on the performance of the compressor with better performing reeds exhibiting smaller displacement and faster response.

The performance of the described compressor could be possibly further enhanced by using different (active) valve and by placing a suction port with a valve in the resonator wall where standing wave has its pressure antinode. Moreover, the shape of the optimized resonator and the corresponding maximum acoustic pressure amplitude inside the resonator very much depend on the constraints used in the optimization procedure. By choosing different constraints (especially the minimum radius $r_{\min }$ ) optimization procedure would produce different resonator shape which could produce higher acoustic pressure amplitude and better performance of the acoustic compressor.

\section{ACKNOWLEDGEMENTS}

This work was supported by GACR grant P101/12/1925.

\section{REFERENCES}

[1] B. Coppens, J. V. Sanders. Finite amplitude standing waves in rigid walled tubes. JASA 52:1024-1034, 1968.

[2] D. B. Cruikshank. Experimental investigation of finite-amplitude acoustic oscillations in a closed tube. JASA 52:1024-1036, 1972.

[3] D. F. Gaitan, A. A. Atchley. Finite amplitude standing waves in harmonic and anharmonic tubes. JASA pp. 2689-2495, 1993

[4] C. Lawrenson, B. Lipkens, T. S. Lucas, et al. Measurements of macrosonic standing waves in oscillating closed cavities. JASA 104:623-636, 1998.

[5] M. Ilgamov, R. Zaripov, R. Galiullin, V. Repin. Nonlinear oscillations of a gas in a tube. Applied Mechanics Reviews 49:137-154, 1996.

[6] Y. A. Ilinskii, B. Lipkens, T. S. Lucas, et al. Nonlinear standing waves in an acoustical resonator. JASA 104:2664-2674, 1998 .

[7] X. Li, J. Finkbeiner, G. Raman, et al. Optimized shapes of oscillating resonators for generating high-amplitude pressure waves. JASA 116:2814-2821, 2004.

[8] M. Červenka, M. Šoltés, M. Bednařík. Optimal shaping of acoustic resonators for the generation of high-amplitude standing waves. JASA 136:1003-1012, 2014.

[9] T. Nakane. Discharge phenomenon in high-intensity acoustic standing wave field. IEEE Trans Plasma Sci.

[10] G. W. Swift. Thermoacoustic engines. JASA 84:1145-1180, 1988 .

[11] A. G. Bodine. Resonant gas compressor and method, 1952. US Patent 2,581,902.

[12] C. Macrosonix. Standing wave compressor, 1991. EP Patent App. EP19,910,301,934.

[13] A. El-Sabbagh. Gas-filled Axisymmetric Acoustic Resonators. VDM Verlag Dr. Muller Aktiengesellschaft \& Co. KG, 2008.

[14] M. Masuda, S. Kawashima. A study on effects of the valves of acoustic compressors on their delivery flow rate. International congress on acoustics Madrid september 2007.

[15] A. Hossain, M. Kawahashi, T. Nagakita, et al. Application of finite amplitude oscillation of air-column in closed tube to design acoustic compressor. Proceeding of 18th International Congress on Acoustics pp. 381-384, 2004 . 